\title{
High-Throughput Quantitative Analysis by Desorption Electrospray Ionization Mass Spectrometry
}

\author{
Nicholas E. Manicke, Thomas Kistler, Demian R. Ifa, R. Graham Cooks, \\ and Zheng Ouyang \\ Department of Chemistry, Purdue University, Bindley Biosciences Center, West Lafayette, Indiana, USA
}

A newly developed high-throughput desorption electrospray ionization (DESI) source was characterized in terms of its performance in quantitative analysis. A 96-sample array, containing pharmaceuticals in various matrices, was analyzed in a single run with a total analysis time of $3 \mathrm{~min}$. These solution-phase samples were examined from a hydrophobic PTFE ink printed on glass. The quantitative accuracy, precision, and limit of detection (LOD) were characterized. Chemical background-free samples of propranolol (PRN) with PRN- $\mathrm{d}_{7}$ as internal standard (IS) and carbamazepine (CBZ) with CBZ-d ${ }_{10}$ as IS were examined. So were two other sample sets consisting of PRN/PRN- $\mathrm{d}_{7}$ at varying concentration in a biological milieu of $10 \%$ urine or porcine brain total lipid extract, total lipid concentration $250 \mathrm{ng} / \mu \mathrm{L}$. The background-free samples, examined in a total analysis time of $1.5 \mathrm{~s} / \mathrm{sample}$, showed good quantitative accuracy and precision, with a relative error (RE) and relative standard deviation (RSD) generally less than 3\% and 5\%, respectively. The samples in urine and the lipid extract required a longer analysis time ( $2.5 \mathrm{~s} /$ sample) and showed RSD values of around $10 \%$ for the samples in urine and 4\% for the lipid extract samples and RE values of less than 3\% for both sets. The LOD for PRN and CBZ when analyzed without chemical background was 10 and 30 $\mathrm{fmol}$, respectively. The LOD of PRN increased to $400 \mathrm{fmol}$ analyzed in $10 \%$ urine, and $200 \mathrm{fmol}$ when analyzed in the brain lipid extract. (J Am Soc Mass Spectrom 2009, 20, 321-325) (C) 2009 Published by Elsevier Inc. on behalf of American Society for Mass Spectrometry

Q uantitative analysis of pharmaceutical compounds in biological fluids is typically carried out using liquid chromatography separation combined with a mass spectrometer fitted with an atmospheric pressure ionization (API) ion source, usually electrospray ionization or atmospheric pressure chemical ionization (LC/ESI-MS or LC/APCI-MS). Such a system can analyze one sample for numerous components in a matter of a few minutes [1]. In most applications, the separation device is directly coupled on-line with the MS. In others, however, extensive prior separation is not necessary, and it can be replaced by a simpler extraction step. In these cases, current highthroughput approaches include direct injection of the sample solution into the MS from a 96-well sample plate for analysis by API [2], or the spotting of solid samples with an organic matrix for analysis by matrix-assisted laser desorption/ionization (MALDI) [3].

New options for the ionization of samples at atmospheric pressure have emerged recently. The family of ambient ionization methods for mass spectrometry,

Address reprint requests to Dr. R. G. Cooks, or Dr. Z. Ouyang, Department of Chemistry, Purdue University, Bindley Biosciences Center, West Lafayette, IN 47907, USA. E-mail: cooks@purdue.edu or E-mail: ouyang@purdue. edu which originated with desorption electrospray ionization (DESI) [4] and direct analysis in real time (DART) [5], has rapidly expanded to include a number of new members including MALDESI, LAESI, PADI, ASAP, DBDI, and ELDI [6-11]. In DESI-MS, samples are analyzed by directing a pneumatically-assisted stream of charged microdroplets at the surface in the ambient environment. The potential utility of DESI in highthroughput analysis is obvious since samples do not need prior preparation or transfer into vacuum, and there are numerous reports in the literature demonstrating rapid sample analysis by DESI [12-15]. Reports of high-throughput analysis in which a large number of samples are analyzed quickly in rapid succession are much fewer [16]. The extension from rapid single-sample analysis to high-throughput is not trivial since a number of issues, such as sample carry-over, sample manipulation, and data acquisition, require consideration. It is therefore desirable to demonstrate the quality achievable in this type of analysis and evaluate the quantitative performance of experiments in which a large number of samples are quickly and efficiently examined in a reproducible fashion. In this report we describe the highthroughput quantitation of 96-sample arrays using a $2 \mathrm{D}$ imaging/high throughput DESI source.
(C) 2009 Published by Elsevier Inc. on behalf of American Society for Mass Spectrometry. $1044-0305 / 09 / \$ 32.00$

doi:10.1016/j.jasms.2008.10.011
Published online November 1, 2008 Received June 23, 2008 Revised October 11, 2008 Accepted October 13, 2008 


\section{Experimental}

\section{Materials}

PRN and CBZ were purchased from Sigma-Aldrich (St. Louis, MO), and the isotopically labeled standards PRN- $\mathrm{d}_{7}$ and CBZ- $\mathrm{d}_{10}$ were acquired from C/D/N Isotopes, Inc. (Pointe-Claire, Quebec, Canada). Glass slides printed with a hydrophobic ink containing PTFE were custom-made by Erie Custom Glass (Portsmouth, NH). Porcine brain total lipid extract was purchased from Avanti Polar Lipids, Inc. (Alabaster, AL).

\section{Sample Preparation}

Four sets of samples were analyzed to provide calibration curves: two without any chemical background, one in $10 \%$ urine (diluted in deionized water), and one in $250 \mathrm{ng} / \mu \mathrm{L}$ brain total lipid extract. The two background-free calibration curves were PRN with PRN- $\mathrm{d}_{7}$ as IS and CBZ with CBZ- $\mathrm{d}_{10}$ as IS. The concentrations of the analyte were $0.05,0.1,0.5,1$, and $5 \mu \mathrm{M}$, and the concentration of IS was $0.5 \mu \mathrm{M}$. All solutions were prepared in 1:1 (vol:vol) methanol: water. For the samples in a biological milieu, PRN was used at concentrations of $0.1,0.5,1$, and $5 \mu \mathrm{M}$ with $1 \mu \mathrm{M}$ PRN-d . $_{7}$

One $\mu \mathrm{L}$ of each sample was deposited on a custommade glass slide with a "96 well" type of format, except that the "wells" were small raised spots (Figure 1). The slides were $124 \mathrm{~mm} \times 83 \mathrm{~mm}$ and were printed with 96 dots using a hydrophobic ink containing PTFE $[17,18]$. The dots were $3.2 \mathrm{~mm}$ in diameter and $9 \mathrm{~mm}$ apart in a regular $12 \times 8$ grid. Five replicates of each concentration for each sample type were deposited for a total of 90 samples. The final six PTFE dots were used for blanks and a spot of ink from a Sharpie pen (Sanford L.P., Oak Brook, IL) for manual calibration of the plate position.

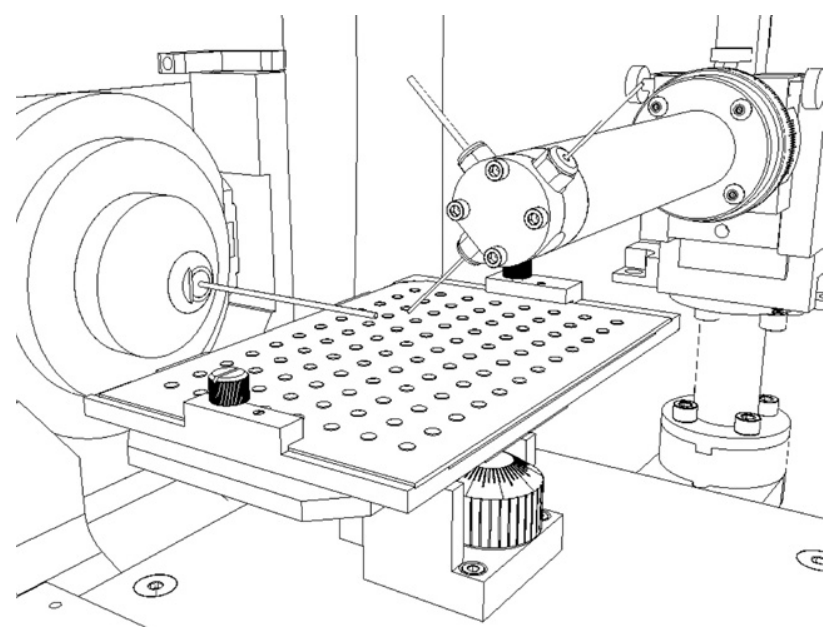

Figure 1. Sketch of the automated moving stage with sample plate.

\section{Sample Analysis}

The 2D DESI-MS stage used in this study was mounted on an X-Y moving table driven by two stepping motors with ca. $10 \mathrm{~cm} \times 10 \mathrm{~cm}$ travel distances. The table, motors, and control components were purchased from Danaher Motion (Westborough, MA). A lab-built DESI source was used [19], and its position and incident angle were adjusted by an XYZ integrated linear stage (Newport, Richmond, CA) and a rotary stage (Parker Automation, Irwin, PA). The stage, table, motors, and source were mounted on a custom-machined apparatus designed to fit a Thermo Scientific LTQ mass spectrometer (San Jose, CA), which was used for all of the experiments.

The 96-sample array analysis was performed in a point-to-point fashion in which the moving stage was paused on each sample for some time and then transitioned to the next sample. The analysis time at each spot was $1 \mathrm{~s}$ for the background free samples and $2 \mathrm{~s}$ for the samples in a biological milieu. The scan time of the mass spectrometer was $290 \mathrm{~ms}$, the maximum injection time was $250 \mathrm{~ms}$, and automatic gain control was on. The moving stage speed was adjusted to make the time lapse between completion of one analysis and commencement of the next $0.5 \mathrm{~s}$.

A DESI spray solvent of 1:1 (vol:vol) methanol:water with a flow rate of $1.5 \mu \mathrm{L} / \mathrm{min}$ was used. Other source parameters have been described elsewhere [19].

\section{Data Analysis}

Three spectra were averaged for each sample and the peak height ratios (PHR) between the analyte and the IS were recorded. This value was averaged for a given sample type and concentration and then used for the calibration or measurement. The calibration data were fitted to a straight line using ordinary least-squares and weighted least-squares with weightings of $1 / x, 1 / x^{2}$, and $1 / \mathrm{s}^{2}$. All of the data presented here is for $1 / x$ weighted least-squares because this minimized the RE. The LOD was defined to be the lowest concentration required to give a signal equal to the average intensity of the blank signal plus three times the standard deviation of the blank.

\section{Results and Discussion}

DESI should be readily applicable to the high-throughput analysis of a large number of dried liquid samples, simply by automatically spotting the samples in some reproducible format. To explore this application, we procured custom-made PTFE printed glass slides in a 96-sample format (Figure 1). This PTFE printed glass substrate has been shown to be an effective and versatile surface for DESI that gives strong, stable signal for a wide array of analytes $[18,20]$. This system offers several advantages. First, reproducibility is maximized because a large number of samples can be analyzed without altering the 
source configuration. Second, the deposition of samples onto this substrate should be easily accomplished using existing robotic spotters. Finally, neither the carryover nor the signal spikes observed in some other studies were seen due to the relatively large distance between the samples and the high speed of the stage [21].

DESI analysis was performed on four types of samples in a 96-sample array in the course of a single experiment: two sample types were free of any chemical background, one was made in a $10 \%$ urine solution, and one in $250 \mathrm{ng} / \mu \mathrm{L}$ brain total lipid extract. Isotopically labeled standards were selected to minimize complicating effects that might arise from the use of chemically similar but distinct standards [18]. The total analysis time per sample (including analysis time and the time required to move between samples) was $1.5 \mathrm{~s}$ for background-free samples and $2.5 \mathrm{~s}$ for the samples in a biological milieu, allowing the entire 96 sample plate to be analyzed in just over $3 \mathrm{~min}$. A portion of the experiment is shown in Figure 2a. The five samples shown contain an increasing amount of PRN and a constant amount of IS. This measurement was repeated five times for each of the four sample sets, resulting in a total of 90 samples. Each of the four sets of samples was subjected to a linear fit and showed acceptable values for correlation coefficient, accuracy, and precision. As an example, a plot of the average PHR against the concentration of the original sample solution for the chemical-background-free PRN samples is shown in Figure $2 b$.

The data obtained during a typical run are shown in Table 1. For the two background-free calibration curves, the LOD was $0.01 \mu \mathrm{M}$ (10 fmol absolute amount) for PRN and $0.03 \mu \mathrm{M}(30 \mathrm{fmol})$ for CBZ. The lowest concentration analyzed for each set of samples $(0.05$ $\mu \mathrm{M})$ was reliably detected with a signal-to-noise ratio of 5 to 10 but was excluded from the linear fit. The analyte/IS ratio at this concentration was larger than the linear fit would suggest and therefore increased the $\mathrm{RE}$ of the other data points significantly. For the four
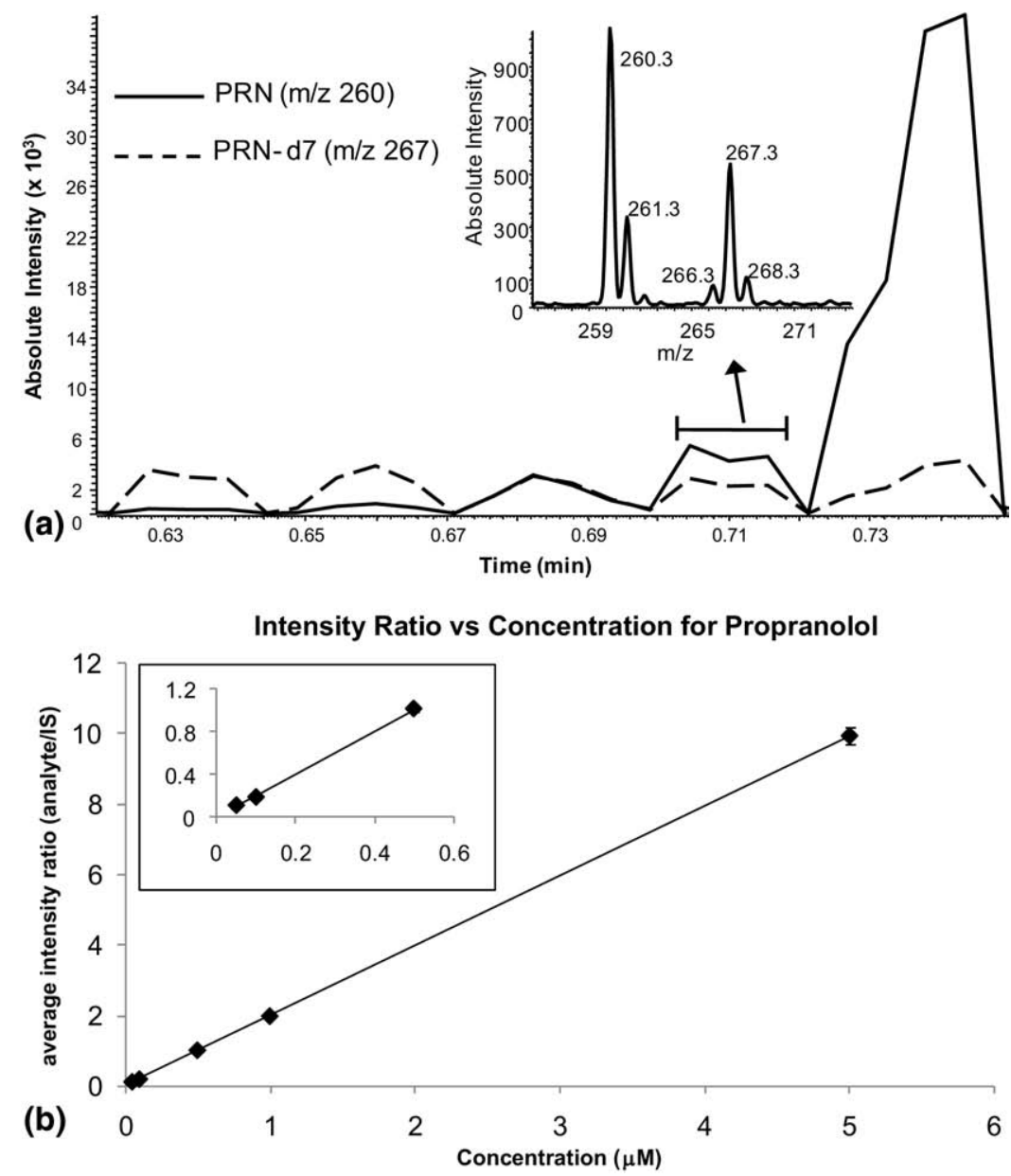

Figure 2. (a) Extracted ion chronograms from ca. 0.60 to 0.75 min during the experiment, showing the analysis of five samples with increasing concentrations of PRN and a constant $0.5 \mu \mathrm{M}$ concentration of PRN- $d_{7}$. Three MS scans from each sample were averaged to obtain the peak height ratio. A representative example spectrum for a $1 \mu \mathrm{M}$ PRN sample is shown in the inset. (b) Average peak height ratio for PRN and PRN- $\mathrm{d}_{7} \pm \mathrm{SD}(N=5)$ versus original solution concentration. The inset shows a closer view of the lower concentration range. 
Table 1. Average analyte/IS ion abundance ratio, RSD, and RE obtained from weighted linear least squares (1/x) for four different calibration samples run on a single 96-sample plate

\begin{tabular}{|c|c|c|c|c|c|c|c|}
\hline & Analyte concentration $(\mu \mathrm{M})$ & 0.05 & 0.1 & 0.5 & 1 & 5 & $y=a x+b$ \\
\hline \multirow[t]{3}{*}{$\begin{array}{l}\text { PRN with } 0.5 \mu \mathrm{M} \text { PRN- } d_{7} \text { IS } \\
1.5 \text { s/sample }\end{array}$} & $\begin{array}{l}\text { Average PHR, } \\
\quad N=5\end{array}$ & 0.118 & 0.19 & 1.01 & 1.98 & 9.9 & $\begin{array}{l}a=1.990 \\
b=0.00\end{array}$ \\
\hline & RSD (\%) & 6.7 & 5.5 & 2.0 & 2.2 & 2.4 & $1.971 \leq \mathrm{a} \leq 2.009^{\mathrm{b}}$ \\
\hline & $\mathrm{RE}(\%)$ & $\mathrm{NI}^{\mathrm{a}}$ & $1.6 \%$ & -2.1 & 0.3 & 0.1 & $-0.05 \leq b \leq .05$ \\
\hline \multirow{3}{*}{$\begin{array}{l}\text { CBZ with } 0.5 \mu \mathrm{M} \mathrm{CBZ-d_{10 }} \text { IS } \\
1.5 \text { s/sample }\end{array}$} & Average PHR, $N=5$ & 0.16 & 0.19 & 0.86 & 1.69 & 8.6 & $a=1.71$ \\
\hline & RSD $(\%)$ & 6.4 & 6.8 & 2.2 & 3.4 & 2.4 & $\mathrm{~b}=0.02$ \\
\hline & RE (\%) & $\mathrm{NI}$ & -3 & 1 & 2 & 0 & $\begin{array}{r}1.67 \leq a \leq 1.76 \\
-0.09 \leq b \leq 0.13\end{array}$ \\
\hline \multirow{3}{*}{$\begin{array}{l}\text { PRN with } 1 \mu \mathrm{M} \text { PRN- } \mathrm{d}_{7} \text { IS } \\
\text { in } 10 \% \text { urine } \\
2.5 \text { s/sample }\end{array}$} & Average PHR, $N=5$ & NA & ND & 0.48 & 0.94 & 4.9 & $\mathrm{a}=0.991$ \\
\hline & RSD $(\%)$ & NA & ND & 8.3 & 11.1 & 9.4 & $b=-0.02$ \\
\hline & $\mathrm{RE}(\%)$ & NA & ND & -2.2 & 2.4 & -0.3 & $\begin{array}{l}0.965 \leq \mathrm{a} \leq 1.017 \\
-0.09 \leq \mathrm{b} \leq .04\end{array}$ \\
\hline \multirow{4}{*}{$\begin{array}{l}\text { PRN with } 1 \mu \mathrm{M} \text { PRN- } \mathrm{d}_{7} \text { IS } \\
\text { in } 250 \mathrm{mg} / \mathrm{mL} \text { brain } \\
\text { extract } \\
2.5 \text { seconds/sample }\end{array}$} & Average PHR, $N=5$ & NA & ND & 0.477 & 0.98 & 5.0 & $a=0.9987$ \\
\hline & RSD (\%) & NA & ND & 1.9 & 3.4 & 4.3 & $b=-0.021$ \\
\hline & RE (\%) & NA & ND & 0.2 & -0.2 & 0.0 & $0.9966 \leq a \leq 1.001$ \\
\hline & & & & & & & $-0.027 \leq b \leq-0.015$ \\
\hline
\end{tabular}

aNA: not analyzed; ND: not detected; NI: not included in the linear fit. b95\% Confidence interval.

remaining concentrations in the two curves, RSDs of just over $5 \%$ were obtained for the lowest concentration and well below $5 \%$ for the remaining points. REs less than $3 \%$ were obtained for all of the concentrations.

Two PRN/PRN- $\mathrm{d}_{7}$ calibration curves were recorded in biological matrices. As expected, the LODs in these biological mixtures were higher than those of the pure samples. In the $10 \%$ urine and brain lipid extract, the LOD was 0.4 and $0.2 \mu \mathrm{M}$, respectively. It should be noted that the LOD of the same sample in urine would be higher since the sample is in $10 \%$ urine rather $100 \%$. But despite the higher LODs, DESI quantitation in these biological mixtures shows acceptable figures of merit, with RSDs generally less than $10 \%$ in the case of the $10 \%$ urine and less than $5 \%$ in brain total lipid extract. The REs are under 5\% for both sample sets. Therefore, while chemical interference affects the LOD and the sensitivity, it does not have a significant effect on the reproducibility or linearity of the analysis in the concentration range studied here. In our experiments, we demonstrated quantitative accuracy and precision with an average analysis time of less than $2 \mathrm{~s} /$ sample. If only qualitative results are desired by DESI, faster analysis times can of course be used, perhaps as little as the time needed to collect a single scan. One limitation to the speed of DESI analysis, however, is the time required to wet the surface before analyte desorption can occur [22].

\section{Conclusions}

Interest in DESI-MS and other ambient ionization methods has been increasing rapidly due in large part to the potential these methods have for high-throughput analysis and automation. We demonstrate the capability of a new DESI source for high throughput analysis; a 96-sample plate was analyzed in about $3 \mathrm{~min}$ with reasonable accuracy, precision, and LODs. During method development, prior separation of the samples will sometimes prove to be unnecessary or can be done off-line. In such cases, DESI-MS will likely give results comparable to MALDI or direct injection ESI, but with a significantly simpler experimental set-up, less complex equipment, and less sample preparation since no matrix is required.

\section{Acknowledgments}

The authors acknowledge support for this work by the National Science Foundation, CHE 0412782, and Thermo Fisher Corporation.

\section{References}

1. Hopfgartner, G.; Bourgogne, E. Quantitative High-Throughput Analysis of Drugs in Biological Matrices by Mass Spectrometry. Mass Spectrom. Rev. 2003, 22(3), 195-214.

2. Dethy, J. M.; Ackermann, B. L.; Delatour, C.; Henion, J. D; Schultz, G. A. Demonstration of Direct Bioanalysis of Drugs in Plasma Using Nanoelectrospray Infusion from a Silicon Chip Coupled with Tandem Mass Spectrometry. Anal. Chem. 2003, 75(4), 805-811.

3. Kovarik, P.; Grivet, C.; Bourgogne, E.; Hopfgartner, G. Method Development Aspects for the Quantitation of Pharmaceutical Compounds in Human Plasma with a Matrix-Assisted Laser Desorption/Ionization Source in the Multiple Reaction Monitoring Mode. Rapid Commun. Mass Spectrom. 2007, 21, 911-919.

4. Takats, Z.; Wiseman, J. M.; Gologan, B.; Cooks, R. G. Mass Spectrometry Sampling Under Ambient Conditions with Desorption Electrospray Ionization. Science 2004, 306(5695), 471-473.

5. Cody, R. B.; Laramee, J. A.; Durst, H. D. Versatile New Ion Source for the Analysis of Materials in Open Air under Ambient Conditions. Anal. Chem. 2005, 77(8), 2297-2302.

6. Sampson, J. S.; Hawkridge, A. M.; Muddiman, D. C. Generation and Detection of Multiply-Charged Peptides and Proteins by MatrixAssisted Laser Desorption Electrospray Ionization (MALDESI) Fourier Transform Ion Cyclotron Resonance Mass Spectrometry. J. Am. Soc. Mass Spectrom. 2006, 17(12), 1712-1716.

7. Nemes, P.; Vertes, A. Laser Ablation Electrospray Ionization for Atmospheric Pressure, in Vivo, and Imaging Mass Spectrometry. Anal. Chem. 2007, 79(21), 8098-8106.

8. Ratcliffe, L. V.; Rutten, F. J. M.; Barret, W. T.; Seymour, D.; Greenwood, C.; Aranda-Gonzalvo, Y.; Robinson, S.; McCoustra, M. Surface Analysis Under Ambient Conditions Using Plasma-Assisted Desorption/Ionization Mass Spectrometry. Anal. Chem. 2007, 79, 6094-6101. 
9. McEwen, C. N.; McKay, R. G.; Larsen, B. S. Analysis of Solids, Liquids, and Biological Tissues Using Solids Probe Introduction at Atmospheric Pressure on Commercial LCS/Ms Instruments. Anal. Chem. 2005, 77(23), 7826-7831.

10. Na, N.; Zhao, M.; Zhang, S.; Yang, C. Development of a Dielectric Barrier Discharge Ion Source for Ambient Mass Spectrometry. J. Am. Soc. Mass Spectrom. 2007, 18, 1859-1862.

11. Shiea, J.; Huang, M.-Z.; Hsu, H.-J.; Lee, C.-Y.; Yuan, C.-H.; Beech, I.; Sunner, J. Electrospray-Assisted Laser Desorption/Ionization Mass Spectrometry for Direct Ambient Analysis of Solids. Rapid Commun. Mass Spectrom. 2005, 19, p 3701-3704.

12. Mulligan, C. C.; MacMillan, D. K.; Noll, R. J.; Cooks, R. G. Fast Analysis of High-Energy Compounds and Agricultural Chemicals in Water with Desorption Electrospray Ionization Mass Spectrometry. Rapid Commun. Mass Spectrom. 2007, 21, 3729-3736.

13. Huang, G.; Chen, H.; Zhang, X.; Cooks, R. G.; Ouyang, Z. Rapid Screening of Anabolic Steroids in Urine by Reactive Desorption Electrospray Ionization. Anal. Chem. 2007, 79, 8327-8332.

14. Williams, J. P.; Hilton, G. R.; Thalassinos, K.; Jackson, A. T.; Scrivens, J. H. The Rapid Characterization of Poly(Ethylene Glycol) Oligomers Using Desorption Electrospray Ionization Tandem Mass Spectrometry Combined with Novel Product Ion Peak Assignment Software. Rapid Commun. Mass Spectrom. 2007, 21(11), 1693-1704.

15. Kauppila, T. J.; Talaty, N.; Kuuranne, T.; Kotiaho, T.; Kostiainen, R.; Cooks, R. G. Rapid Analysis of Metabolites and Drugs of Abuse from Urine Samples by Desorption Electrospray Ionization-Mass Spectrometry. Analyst 2007, 132(9), 868-875.
16. Chen, H.; Talaty, N.; Takats, Z.; Cooks, R. G. Desorption Electrospray Ionization Mass Spectrometry for High Throughput Analysis of Pharmaceutical Samples in the Ambient Environment. Anal. Chem. 2005, 77, 6915-6927.

17. Pasilis, S. P.; Kertesz, V.; Van Berkel, G. J. Surface Scanning Analysis of Planar Arrays of Analytes with Desorption Electrospray IonizationMass Spectrometry. Anal. Chem. 2007, 79, 5956-5962.

18. Ifa, D. R.; Manicke, N. E.; Rusine, A. L.; Cooks, R. G. Quantitative Analysis of Small Molecules by Desorption Electrospray Ionization Mass Spectrometry from Polytetrafluoroethylene Surfaces. Rapid Commun. Mass Spectrom 2008, 22(4), 503-510.

19. Ifa, D. R. Wiseman, J. M. Song Q. Y. Cooks, R. G. Development of Capabilities for Imaging Mass Spectrometry under Ambient Conditions with Desorption Electrospray Ionization (DESI). Int. J. Mass Spectrom 2007, 259, 8-15.

20. Manicke, N. E.; Wiseman, J. M.; Ifa, D. R.; Cooks, R .G. Desorption Electrospray Ionization (DESI) Mass Spectrometry and Tandem Mass Spectrometry (MS/MS) of Phospholipids and Sphingolipids: Ionization, Adduct Formation, and Fragmentation. J. Am. Soc. Mass Spectrom. 2008, 19(4), 531-543.

21. Kertesz, V.; van Berkel, G. J. Scanning and Surface Alignment Considerations in Chemical Imaging with Desorption Electrospray Mass Spectrometry. Anal. Chem. 2008, 80, 1027-1032.

22. Bereman, M. S.; Muddiman, D. C. Detection of Attomole Amounts of Analyte by Desorption Electrospray Ionization Mass Spectrometry (DESI-MS) Determined Using Fluorescence Spectroscopy. J. Am. Soc. Mass Spectrom. 2007, 18, 1093-1096. 